\title{
Uso de recursos digitais 3D no ensino de química: as potencialidades do Geogebra ${ }^{\circledR}$
}

\author{
Uso de los recursos digitales $3 D$ en la enseñanza de la química: el \\ potencial del Geogebra®
}

The use of $3 D$ digital resources in chemistry teaching: the potential of Geogebra®

\author{
José Oxlei de Souza Ortiz ${ }^{1}$ \\ Willian Daniel Pessoa ${ }^{2}$ \\ Aline Machado Dorneles ${ }^{3}$
}

\begin{abstract}
Resumo
O presente artigo busca apresentar as potencialidades do aplicativo Geogebra ${ }^{\circledR}$ no ensino de Química. A intenção com o estudo é de fomentar o uso de recursos digitais na formação de professores de Química, bem como, auxiliar na construção do conhecimento químico, por meio da visualização, percepção e principalmente na construção geométrica molecular de algumas ligações químicas. As transformações químicas podem ser compreendidas no nível macroscópico (observação do fenômeno), mas há uma dificuldade em transferir os aspectos observáveis no nível macroscópico para o nível microscópico, dificultando a construção de modelos explicativos que se aproximem dos modelos científicos. Dessa forma, entende-se a relevância do o aplicativo Geogebra ${ }^{\circledR}$ na construção do conhecimento em nível microscópico. A análise das potencialidades do aplicativo será orientada pelos fundamentos do design, visando apontar os aspectos relevantes e as potencialidades do recurso digital como ferramenta pedagógica na sala de aula.
\end{abstract}

Palavras-Chave: GeoGebra®; Química; Geometria Molecular; Recursos Digitais; Design.

\section{Resumen}

El presente artículo busca presentar las potencialidades de la aplicación Geogebra ${ }^{\circledR}$ en la enseñanza de Química. La intención con el estudio es de fomentar el uso de recursos digitales en la formación de profesores de Química, así como, auxiliar en la construcción del conocimiento químico, por medio de la visualización, percepción y principalmente en la construcción geométrica molecular de algunas conexiones químicas. Las transformaciones químicas pueden ser comprendidas en el nivel macroscópico (observación del fenómeno), pero hay una dificultad en transferir los aspectos observables en el nivel macroscópico al nivel microscópico, dificultando la construcción de modelos explicativos que se aproximen a los modelos científicos. El análisis de

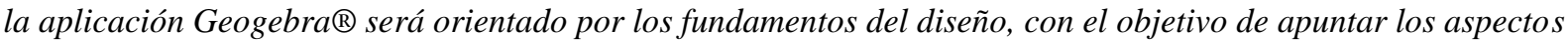
relevantes y las potencialidades del recurso digital como herramienta pedagógica en el aula.

Palabras claves: GeoGebra®, Química; Geometría Molecular; Recursos Digitales; Diseño.

\footnotetext{
${ }^{1}$ Mestrando em Educação em Ciências; Universidade Federal do Rio Grande; Rio Grande, RS, Brasil; joseoxlei@gmail.com.

${ }_{2}$ Mestrando em Educação em Ciências; Universidade Federal do Rio Grande; Rio Grande, RS, Brasil; biomedicowillianpessoa@gmail.com.

${ }^{3}$ Doutora em Educação em Ciências. Professora Adjunta da Escola de Química e Alimentos e do Programa de Pós-Graduação em Educação em Ciências na Universidade Federal do Rio Grande (FURG).

Coordenadora do curso de Química Licenciatura da FURG. End: Av. Itália, Km. 8 s/n - Campus Carreiros, Rio Grande - RS. Email: lidorneles26@gmail.com.
} 


\begin{abstract}
This paper seeks to present the potential of the Geogebra® application in Chemistry teaching. The purpose of this study is to promote the use of digital resources in the training of chemistry teachers, as well as to assist in the construction of chemical knowledge, through visualization, perception and mainly in the molecular geometric construction of some chemical bonds. The chemical transformations can be understood at the macroscopic level (observation of the phenomenon), but there is a difficulty in transferring the observable aspects at the macroscopic level to the microscopic level, making it difficult to construct explanatory models that approach the scientific models. In this way, it is understood the relevance of the application Geogebra® in the construction of knowledge at a microscopic level. The analysis of the potential of the application will be guided by the fundamentals of design, aiming to point out the relevant aspects and the potentialities of the digital resource as a pedagogical tool in the classroom.
\end{abstract}

Keywords: GeoGebra®, Chemistry; Molecular Geometry; Digital Resources; Design.

\title{
1. Introdução
}

O uso das tecnologias digitais está permeando a sala de aula a um nível cada vez mais intenso, desde os projetores, lousas digitais, computadores, laptops e vai até o uso dos smartphones pelos estudantes e professores. Por isso surge a seguinte pergunta, porque não explorar e utilizar essas tecnologias digitais para potencializar o ensino de química na formação de professores? Analisando de fato essa tendência acelerada dos avanços tecnológicos em diversas áreas, precisamos considerar um fator diferencial para a educação: a apropriação de ferramentas digitais empregadas em diversas áreas, que podem se tornar um fator diferencial nos processos de construção do conhecimento professor/estudante e que as novas gerações estão cada vez mais inseridas neste meio.

Ao pensar no uso dessas ferramentas em sala de aula, almejamos dinamizar o processo de aprendizagem, tornando-o mais criativo e dinâmico. Dessa forma, o objetivo do presente trabalho é apresentar as potencialidades do aplicativo GeoGebra $\AA^{4}$ em aumentar a percepção, visualização e, principalmente, a construção da geometria molecular de algumas ligações químicas. O GeoGebra® a princípio foi concebido para a matemática, mas tem uma possível aplicação para a construção e representação de um conteúdo específico para os cursos de química, o qual se caracteriza em melhorar a percepção das ligações moleculares, em sua grande maioria, são representadas no modo tradicional por meio de imagens ou desenhos feitos nos quadros, ou demonstrados em revistas, livros ou mesmo passados em projetores.

Posto isso, o que será apresentado a seguir, é uma analise inicial do aplicativo, a partir dos fundamentos do design, que consiste na percepção e modo de análise do design, para evidenciar os pontos positivos desse recurso digital, no que se refere principalmente o uso como opção de ferramenta pedagógica em sala de aula de Química. Portanto, compreendemos que o uso do recurso digital no processo de criação e construção das ligações químicas em

\footnotetext{
${ }^{4}$ Download realizado no link https://www.geogebra.org/download. Acesso em: 15 set. de 2017.
} 
3D, auxilia na construção do conhecimento microscópico, como também no pensamento criativo e reflexivo (VALENTE, 1999).

\section{O processo de aprendizagem com o auxílio dos recursos digitais na sala de aula}

A educação é um sistema vivo que deve ser reconstruindo a todo instante, principalmente nesse novo contexto tecnológico, onde tudo está acelerado, onde as atualizações acontecem cada vez mais constantes e em curto prazo, poderíamos assim dizer quase todos os dias. Desse modo, será um desafio para educadores e educando criar laços cada vez mais robustos e conectados entre conhecimento e tecnologia, conteúdo e contexto. Sendo assim segundo Moraes (2003, p. 168).

O desafio é ainda maior quando percebemos que as novas bases epistemológicas trazidas pelas teorias físicas e biológicas nos confirmam que a aprendizagem já não pode ser explicada como fenômenos de mudança de conduta que ocorre a partir de informações instrutivas captadas do meio ambiente, pois o que passa com o indivíduo depende de sua estrutura, depende da dinâmica interacional entre o indivíduo e o meio, das circunstância em que o aprendiz encontra-se inserido e que modifica o fluir de suas emoções e de seus pensamentos.

Desse modo, os recursos digitais podem ser aliados na constante reconstrução do ensino, sendo um campo de possibilidades que emerge nesse mundo complexo educacional, tendo um papel de planejar e elaborar artifícios para qualquer área do conhecimento, pela sua capacidade de dialogar em algum nível com quase todos os outros campos do conhecimento. Logo, os diferentes níveis educacionais necessitam ampliar ações interdisciplinares e multidisciplinares entre as áreas do conhecimento, para assim partilhar experiências e tornar mais plena em seu funcionamento.

Considerando o que foi posto, será importante observar com mais cuidados o ser que aprende e também o que ensina, pois durante o ato educacional ocorrem processos cognitivos centrados na ação e reflexão, e que esses atos estão intimamente ligados ao cérebro humano, por isso entendemos relevante o estudo da neurociência cognitiva no processo de aprendizagem, elementos estes que serão apresentados como um suporte teórico para embasar o uso dos recursos tecnológicos na sala de aula, e assim compreender alguns aspectos cognitivos que ocorrem na aprendizagem e no uso das tecnologias, principalmente as digitais. Segundo Simões et al (2016, p. 33).

O objeto das Neurociências cognitivas é o conhecimento do funcionamento cerebral subjacente à cognição. Cognição, segundo a etimologia, origina-se do Latim 
Cognitio onis, que significa origem, aquisição de conhecimento, percepção. É, portanto, a capacidade de adquirir conhecimentos por meio da aprendizagem. Chamamos de cognição todos os processos mentais relacionados a: atenção, memória, linguagem e funções executivas.

Por isso, foi elaborada uma ligação da neurociência com o design emocional (2008), empunhado pelo psicólogo Donald Norman (2008), no qual, está intimamente ligada com a percepção. Temos 3 níveis distintos do design emocional, sendo que cada nível abrangem um modo de percepção, temos assim, o nível visceral, que permeia os sentidos, como: visão, os cheiros, os sons e dentre outros, este nível abrange o plano mais superficial do nosso modo de percepção.

Figura 1: Níveis do design emocional.

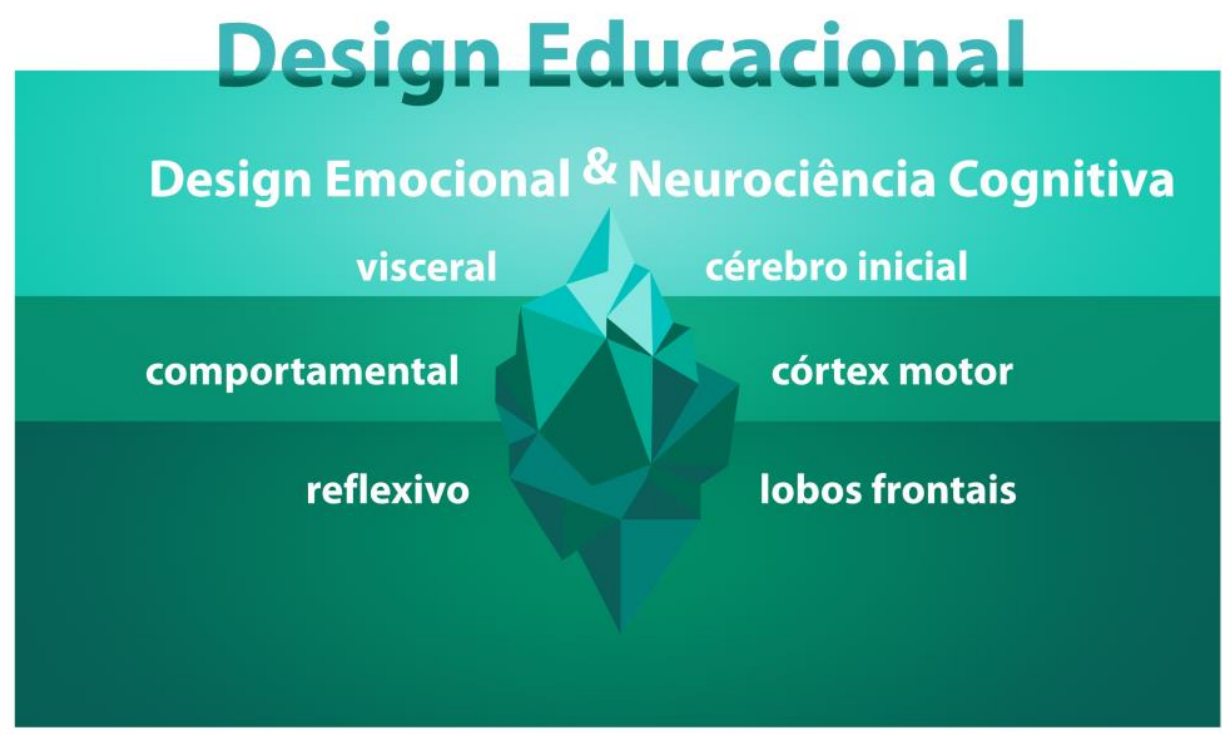

Fonte: autores

Portanto o design visceral está preocupado mais com a forma e a estética, durante o processo de percepção e criação. Já no segundo nível, temos o nível comportamental, no qual atinge o nosso modo de ser e principalmente como nos relacionamos com o objeto experiência, neste nível temos a relação entre o modo como os objetos são manuseados durante o cotidiano e sua concepção para o uso, sendo assim, explorando as funcionalidade e potencialidades de experienciação. No terceiro nível temos o reflexivo, sendo o nível mais abrangente, no qual está intimamente ligado como o modo no qual nos relacionamos com a satisfação pessoal, com nossas lembranças e auto-imagem. No nível reflexivo temos a criação, portanto nesse nível nos utilizamos das inúmeras necessidades dos estudantes, para atingirmos os vários aspectos cognitivos, emocionais e comportamentais. Ao compreender o aspecto desse nível, leva-nos a compreender a importância do nível reflexivo no processo de 
criação e principalmente o da aprendizagem, como nos alerta, Norman (2008) que o valor reflexivo supera as dificuldades comportamentais.

No ensino de Química, por exemplo, no estudo da constituição da matéria, do átomo e das ligações químicas o nível visceral é percebido quando buscamos representar de maneira simplificada, muitas vezes, somente na observação dos fenômenos no macroscópico, por meio do quadro branco, slides, imagens de livros e revistas. No entanto, esse processo fica limitado, há uma dificuldade em compreender o conhecimento abstrato e microscópico, de imaginar e perceber a movimentação espacial dos átomos e moléculas, por isso o uso do recurso digital 3D poderá auxiliar na construção de modelos explicativos que se aproximem dos modelos científicos. Quando o professor utiliza o quadro ou apresentação de slides, estimula os estudantes aos níveis viscerais e comportamentais, mas quando propõe construir ou desenhar as moléculas em um ambiente 3D virtual, no caso o GeoGebra®, estará assim, realizando um exercício cognitivo mais complexo exigindo uma capacidade reflexiva do estudante, principalmente de como ele vai organizar essa informação no espaço tridimensional.

\section{Apresentação do aplicativo GeoGebra ${ }^{\circledR}$ e suas potencialidades no ensino de ligações químicas}

O aplicativo GeoGebra ${ }^{\circledR}$ é um programa de software livre, que pode ser realizado o download e instalado gratuitamente em diversos sistemas operacionais, e o aplicativo ainda tem uma versão web que pode ser utilizado virtualmente na rede de internet, sem precisar instalar no dispositivo. Com isso, se torna um instrumento importante para a educação, e as diversas áreas do conhecimento. Convém mencionar que este software foi idealizado por Markus Hohenwarter, em 2001, com a finalidade de utilizar em escolas para educação matemática (BORGES NETO, 2009, p. 26). No entanto, devido ao seu caráter educativo e dinâmico, ele pode ser utilizado praticamente em todos os níveis de ensino, que combina geometria, álgebra, entre outros, numa mesma aplicação. "É atualmente um dos softwares educativos de matemática mais utilizados no mundo" (NÓBRIGA, et al., 2012, p.2).

Devido as múltiplas funcionalidades do GeoGebra ${ }^{\circledR}$ para o processo de aprendizagem, apresentamos as possibilidades de aplicação no ensino de química e na formação de professores. Apontamos sua relevância no estudo das ligações químicas e na construção da estrutura molecular de diversas substâncias. É possível construir modelos para exibir as ligações químicas em formato 3D, a fim de compreender como estas moléculas se organizam no espaço, possibilitando ao estudante e o professor desenvolver suas próprias hipóteses e tentar verificar se elas são válidas. 
Com isso destacamos seu fácil manuseio onde existe uma interface deste, que é constituída de uma janela gráfica que se divide em uma área de desenho, uma janela de álgebra e um campo de entrada de comandos. A área de desenho possui um sistema de eixos cartesianos onde o usuário faz as construções geométricas com o mouse. Ao mesmo tempo as coordenadas e equações correspondentes são mostradas na janela de álgebra. O campo de entrada de comandos é usado para escrever coordenadas, equações, comandos e funções, e estes são mostrados na área de desenho imediatamente após pressionar a tecla "Enter".

A partir desta janela podemos desenvolver tarefas combinadas para um melhor aproveitamento e entendimento do conteúdo. Neste momento, procuramos fazer adequações para explorar o quadro de recursos oferecido e construímos as ligações entre os átomos que serão apresentados posteriormente com uma breve descrição sobre os elementos. No primeiro exemplo, apresentamos a molécula da água (figura 2) visualizada em $2 \mathrm{D}$ e 3D, onde possibilita ao estudante um maior entendimento e compreensão sobre como se organiza no espaço, angulação ilustrada da ligação entre os átomos e possíveis ligações com outros átomos.

Figura 2 - Interface gráfica do GeoGebra ${ }^{\circledR}$ e modelo 3D da molécula da água

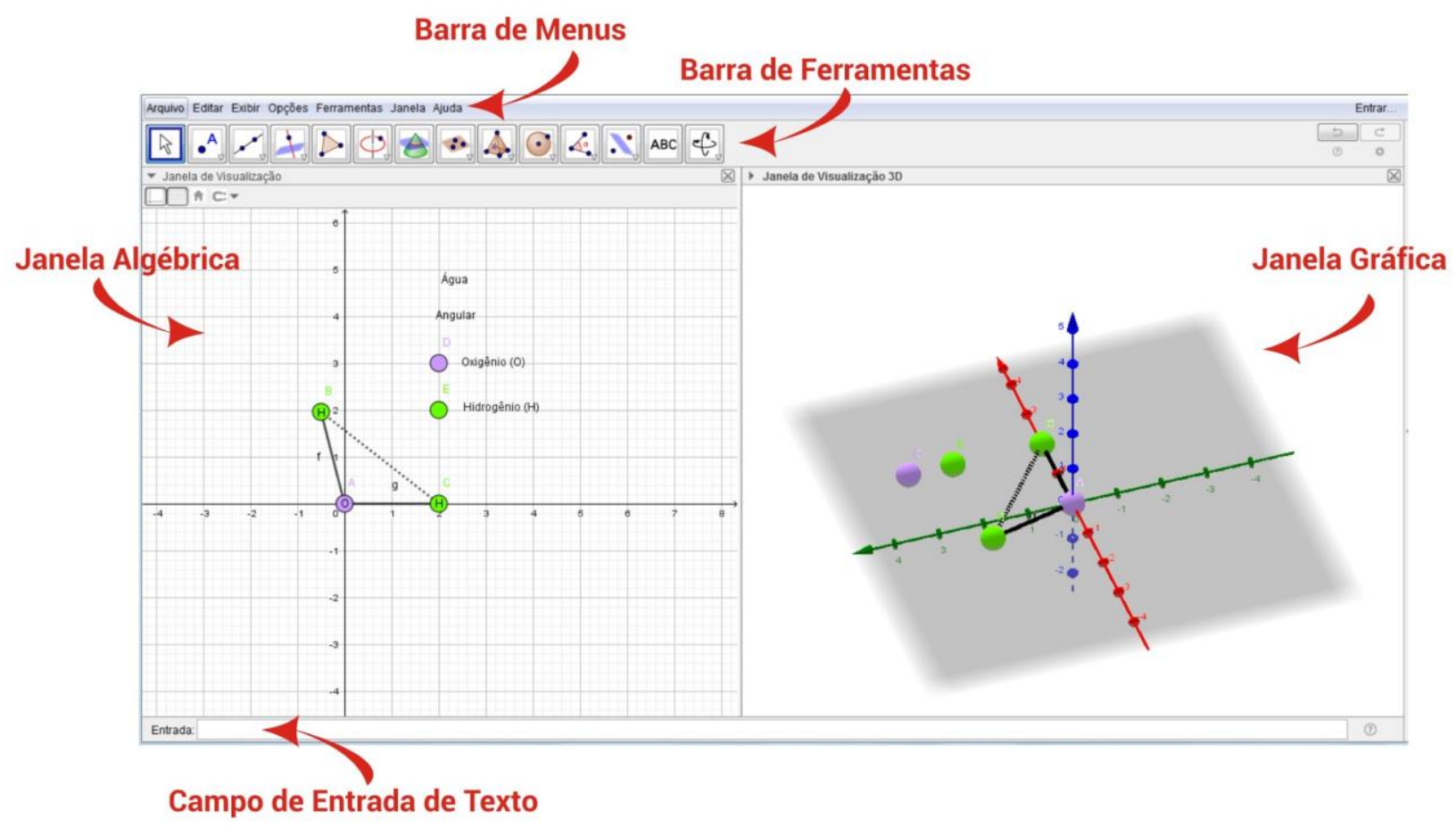

Fonte: Os autores 
$\mathrm{Na}$ figura 3 apresentamos um esquema de representação do gás fluorídrico experimento 1, onde são representados os átomos de acordo com as cores apresentadas. Podemos perceber também que, para facilitar o processo de identificação de cada átomo foi possível inserir a letra que representa cada elemento envolvido na construção da molécula. Para identificação da ligação entre esses átomos foi utilizado um traço simples que representa uma ligação simples.

$\mathrm{Na}$ segunda parte da figura $3 \mathrm{O}$ experimento 2 segue o mesmo padrão dos diversos exemplos apresentados neste trabalho, porém com algumas limitações. Percebemos que não era possível inserir duas linhas entre os átomos para representar uma ligação dupla. Verificamos as possibilidades no software para solucionar este problema, e percebemos que o mesmo oferece uma representação básica de como ilustrar duplas ligações, representado por dois traços que cortam a linha que liga um átomo ao outro. Também é possível representar em esquema de tripla ligação, inserindo três traços nesta mesma linha de ligação.

Figura 3 - Molécula do gás fluorídrico representada no esquema 2D e 3D e molécula do gás carbônico representada no esquema 2D e 3D no GeoGebra®

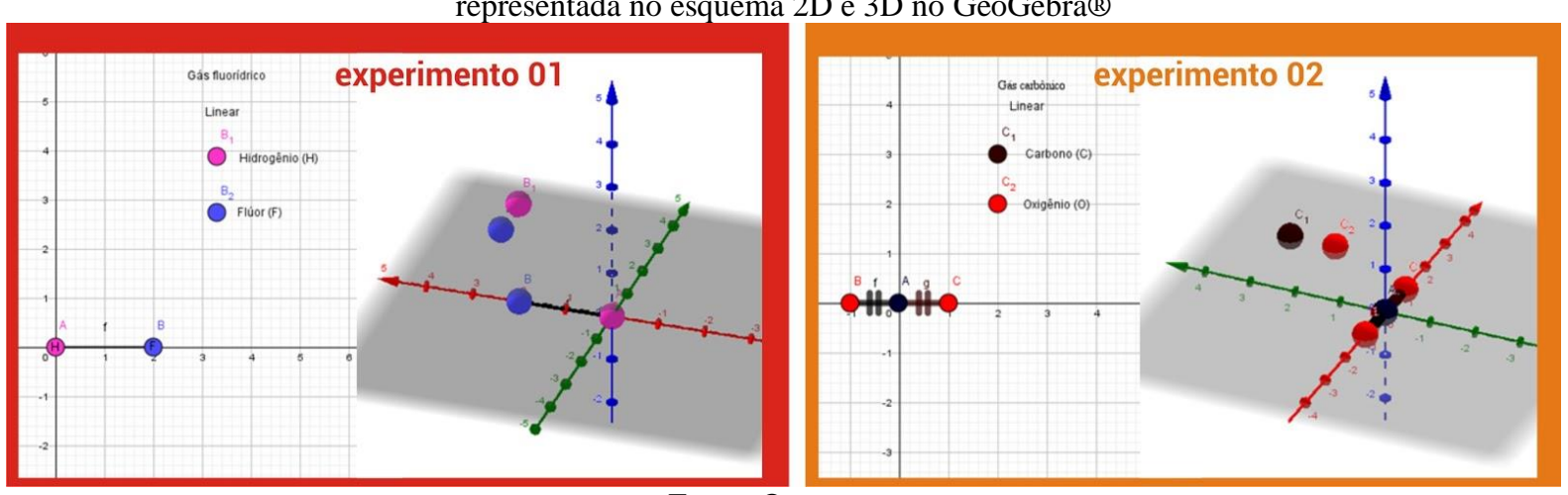

Fonte: Os autores

Para sugestões finais de construção de moléculas, na figura 4 foram apresentados vários exemplos de representação geométrica de moléculas, como por exemplo, piramidal, tetraédrica, angular, entre outras, o que mostra um potencial interessante utilizando este software como ferramenta auxiliar na construção do conhecimento. 
Figura 4 - Experimento 3, molécula da amônia experimento 4, molécula de tetracloreto de carbono experimento 5, molécula de metano representada no esquema 2D e 3D no GeoGebra ${ }^{\circledR}$

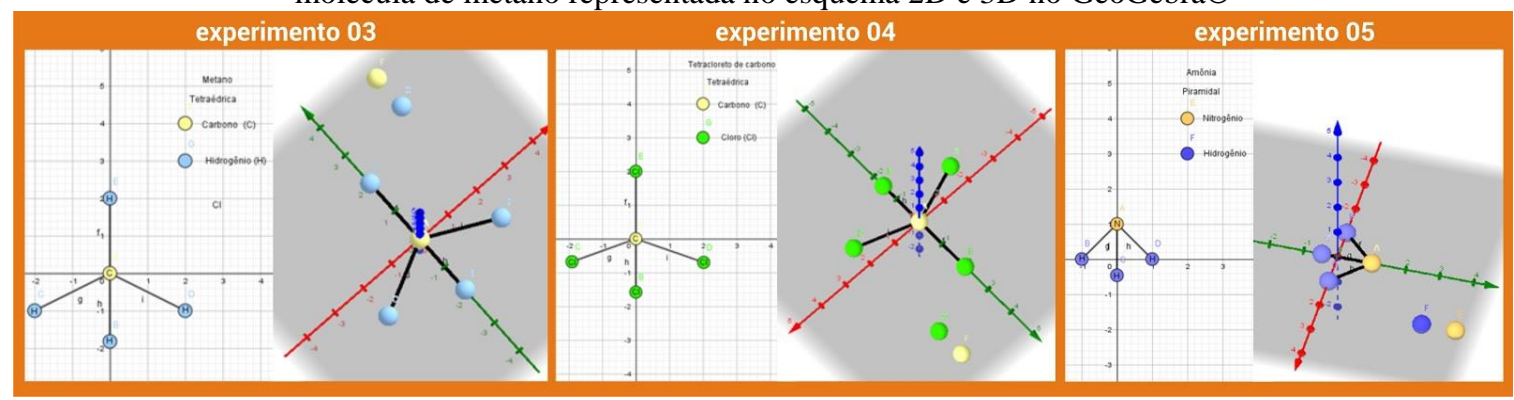

Fonte: Os autores

\section{Considerações finais}

A educação do século XXI estará permeada pelas tecnologias digitais, as contribuições do mundo digital podem ser aplicadas a diversas áreas, principalmente no ensino, com isso, a utilização de ambientes digitais possibilitará um processo de construção ativa, enfatizando a importância por parte do estudante durante o processo de aprendizagem, tornando assim uma ação inseparável do fazer, não somente uma recepção passiva do conhecimento por parte do mesmo, e sim um modo ativo de ser em sala de aula. As contribuições do mundo digital podem ser aplicadas a diversas áreas, por isso teria uma importância mais especificamente para uma informática educativa, por isso, (PELLANDA, 2009, p. 64) “o espaço digital é de uma plasticidade incrível e, por isso, o sujeito vai sendo auto-desafiado para invenções contínuas".

O presente artigo buscou demonstrar como ferramentas auxiliares desenvolvidas para os meios digitais, podem se tornar fundamental na construção do conhecimento. Visamos reforçar que as diversas áreas do conhecimento, principalmente a educação, deve se apossar das tecnologias que surgem a todo o momento, a fim de criar uma versatilidade e dinamismos dentro e fora da sala de aula. O GeoGebra ${ }^{\circledR}$ é uma destas tecnologias que permite muitas outras possibilidades que vão além da representação molecular, nosso propósito inicial aponta caminhos para explorar as diversas potencialidades do aplicativo e de outros recursos digitais no ensino de Química.

Cabe mencionar, a partir da análise do aplicativo que, o software pode apresentar algumas limitações na representação de um maior agrupamento de átomos, logo de moléculas maiores e mais complexas. Mas, destacamos sua relevância para entendimento inicial dos modelos de ligações química. Por fim, argumentamos que o uso do software de fácil manipulação, o GeoGebra ${ }^{\circledR}$, apresenta potencialidades para além do ensino da matemática, para o qual foi criado, mas também para ilustrar diversos esquemas de maneira simples e de 
fácil entendimento para complementar a apresentação do conteúdo de química, tornando um ambiente mais dinâmicas e interativo.

\section{Referências}

BORGES NETO, H. et.al. Manual do Geogebra. Disponível em:

http://ftp.multimeios.ufc.br/ geomeios/geogebra/manual.htm, 2009. Acesso em: 09 out. 2017.

NÓBRIGA, J. C. C. et. al. GGBOOK: uma interface que integrará os ambientes de texto e gráficos no GeoGebra. Revista do Instituto GeoGebra Internacional de São Paulo, São Paulo, v. 1, n. 1, p. 03 - 12, 2012. Disponível em:

http://educonse.com.br/2012/eixo_06/PDF/122.pdf. Acesso em: 08 out. 2017.

MORAES, M. C. Educar na biologia do amor e da solidariedade - Petrópolis, RJ: Vozes, 2003.

NORMAN, D. A. Design emocional. Rio de Janeiro: Editora Rocco, 2008.

PELLANDA, N. M. C. Maturana \& a Educação. Belo Horizonte: Autêntica Editora, 2009.

SIMÕES, E. M. S.; NOGARO, A. Neurociências cognitiva para educadores: aprendizagem e práticas docente no século XXI. Curitiba: Editora CRV, 2016.

VALENTE, J. A. (Org). O computador na sociedade do conhecimento. Campinas:

UNICAMP/NIED, 1999. 